\title{
Selective Dimerization of 1,6-Diynes Catalyzed by Ionic Liquid- Supported Nickel Complexes in An Ionic Liquid/Toluene Biphasic System
}

\author{
Avijit Goswami, ${ }^{a}$ Taichi Ito, ${ }^{a}$ Naoko Saino, ${ }^{b}$ Kouki Kase, ${ }^{a}$ Chikashi Matsuno ${ }^{a}$ and Sentaro Okamoto ${ }^{*}$
}

Received (in $X X X, X X X)$ Xth $X X X X X X X X X 200 X$, Accepted Xth $X X X X X X X X X 200 X$

First published on the web $X t h X X X X X X X X X 200 X$

DOI: 10.1039/b000000x

\begin{abstract}
A $\mathrm{NiCl}_{2}-6 \mathrm{H}_{2} \mathrm{O} / \mathrm{Zn}$ reagent with a 2-aryliminomethylpyridine ligand catalyzed cyclization/polymerization of 1,6-diynes mainly 10 to yield the corresponding poly(1,3-butadienylene) compounds, whereas, $\mathrm{NiBr}_{2}-3 \mathrm{H}_{2} \mathrm{O} / \mathrm{Zn}$ with use of 3 -(2-(2-methoxyethoxy)ethyl)-1-methyl-1H-imidazol-3-ium bromide as a ligand converted 1,6-diynes to the corresponding annulated 1,3,5,7cyclooctatetraenes in a biphasic solvent system consisting of ${ }_{5}$ toluene and an ionic liquid.
\end{abstract}

For homogeneous metal-catalyzed reactions, a bimetallic or bimolecular cooperative mechanism has often been proposed, where a dimerization of the metal species or dimeric interaction of the metal species plays a critical role: ${ }^{1}$ Many cases have been 20 postulated in which a bimetallic or bimolecular mechanism is involved as a major course for effective, selective production of the desired compound. Meanwhile, the reverse case has also been reported in which dimerization of the metal species deactivates the catalyst by forming stable, coordinatively saturated, inert 25 complexes and furthers decomposition by disproportionation. In this context, isolating the catalyst to a solid phase from a solution phase by immobilization onto polymeric supports or inorganic materials is a versatile means not only for avoiding the formation of dimeric metal molecules thereby deactivating and 30 decomposing the catalyst but also for increasing the catalyst reactivity and selectivity via a bimetallic or bimolecular mechanism. $^{2}$

Herein we propose a new strategy for controling the reactions which proceed through formation of a dimeric metal 35 intermediate: Isolation (or immobilization) of the metal-catalyst into an ionic liquid (IL) ${ }^{3}$ phase in an IL/usual organic solvent biphasic system which can separate the catalyst from the employed substrate and maintain a high catalyst concentration in the IL phase throughout the reaction, a situation which might be

40 favorable for reactions via a dimeric metal intermediate and increase the reaction efficiency and/or selectivity (Figure 1).

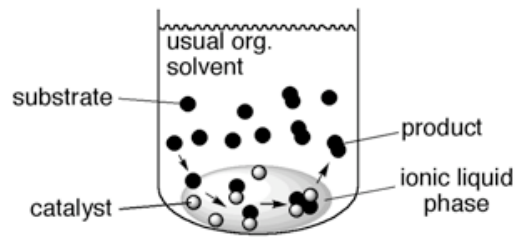

Fig. 1 Illustration of an ionic liquid/usual solvent biphasic system.

Reppe et al. reported a pioneering work for the tetramerization 45 of acetylene giving cyclooctatetraene $(\mathrm{COT})^{4}$ and Wilke suggested a mechanism for the nickel-catalyzed $[2+2+2+2]$ cycloaddition of acetylenes, ${ }^{5}$ which involves the reaction between two nickelacycle complexes derived from two acetylene and the nickel catalyst. Quite recently, Wender and co-workers have so reported a selective dimerization reaction of $\alpha, \omega$-diynes to the corresponding COTs catalyzed by a $(\mathrm{DME}) \mathrm{NiBr}_{2} / \mathrm{Zn}$ [DME $=$ 1,2-dimethoxyethane] reagent in THF- $\mathrm{H}_{2} \mathrm{O}$, where reaction with a large amount of catalyst loading (20 40 mol\%) realized highly selective production of COTs against co-production of $[2+2+2]$ ${ }_{55}$ cycloadducts. ${ }^{6}$ The results were rationalized by assuming the dimeric mechanism that Wilke suggested.

Cyclooctatetraenes (COTs) are of interest as ligands capable of coordinating to various metal atoms and electron transporting components in organic light-emitting devices, and especially as ${ }_{60}$ precursors for polyacetylenes in the ring-opening metathesis polymerization (ROMP) reaction. ${ }^{7}$ Efforts, therefore, have been made in developing methods for synthesizing COTs. ${ }^{8}$ Of the existing methods, the transition metal-catalyzed $[2+2+2+2]$ cycloaddition of alkynes can be considered one of the most 65 attractive and direct.

During the course of studying the transition metal-catalyzed cycloisomerization of alkynes, ${ }^{9}$ we found that a 2(aryliminomethyl)pyridine/ $\mathrm{NiCl}_{2}-6 \mathrm{H}_{2} \mathrm{O} / \mathrm{Zn}$ reagent catalyzed cycloaddition of diethyl dipropargylmalonate (1a) in THF at 70 room temperature to provide the corresponding bis-annulated cyclooctatetraene $\mathbf{2 a}$ in $\sim 30 \%$ yield with $[2+2+2]$ adducts such as dimer 3a and trimer $\mathbf{4 a}$ and a certain amount of polymeric (or oligomeric) molecule(s) as by-products (Scheme 1, run 1 in Table 1). Given our interest in the property of polyene compounds of 75 type $\mathbf{5}$, we investigated the diyne-cyclodimerization to determine the practical conditions required to achieve a production of pure COTs 2 with reasonably low catalyst loading.

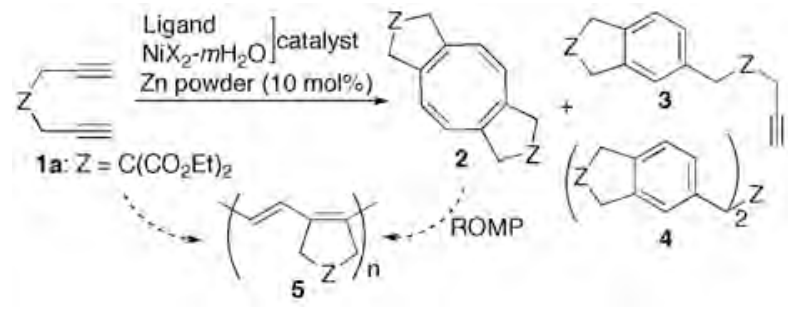

Scheme 1 Nickel-catalyzed cycloaddition of $\alpha, \omega$-diynes.

80 Table 1 shows the results of the reaction of $\alpha, \alpha$ dipropargylmalonate 1a (diethyl ester) and $\mathbf{1 b}$ (dimethyl ester) in the presence of a nickel catalyst $(2 \sim 20 \mathrm{~mol} \%)$ and $\mathrm{Zn}$ powder (4 40 mol\%) under various reaction conditions. Run 1-8 shows the results of the reactions in mono-phasic systems 85 using usual organic solvents. In addition to the aforementioned results of the catalysis with an iminomethylpyridine ligand (DIPIMP) (run 1-3), a $\mathrm{NiCl}_{2}$ $6 \mathrm{H}_{2} \mathrm{O} / \mathrm{Zn}$ reagent without ligand catalyzed the dimerization of 1a in DMF and provided COT 2a in better yield (run 4). The 90 reaction with $5 \mathrm{~mol} \%$ of (DME) $\mathrm{NiBr}_{2}$ in THF resulted in low yield of $2 \mathbf{a}$ (run 5). As reported, ${ }^{6}$ the reaction with increased amount of (DME) $\mathrm{NiBr}_{2}(20 \mathrm{~mol} \%)$ in THF gave $\mathbf{2 a}$ and $\mathbf{2} \mathbf{b}$ in 
Table $\mathbf{1}^{a}$ The reaction of diyne $\mathbf{1}$

\begin{tabular}{|c|c|c|c|c|c|}
\hline Run & Ligand $^{b}$ & $\mathrm{Ni}(\mathrm{mol} \%)$ & Solvent $\left({ }^{\circ} \mathrm{C} / \mathrm{h}\right)$ & Yield of $\mathbf{2}, \%^{c}$ & $\begin{array}{c}\text { Yield } \\
\text { Benzenes }{ }^{d} \text { Polymer }\end{array}$ \\
\hline 1 & DIPIMP & $\mathrm{NiCl}_{2}-6 \mathrm{H}_{2} \mathrm{O}(5)$ & THF (20/12) & $\mathbf{2 a}: 29(17)^{f}$ & $54 \%$ \\
\hline 2 & DIPIMP & $\mathrm{NiCl}_{2}-6 \mathrm{H}_{2} \mathrm{O}(5)$ & NMP (20/12) & $\mathbf{2 a}: 31$ & n.d. (not determined) \\
\hline 3 & DIPIMP & $\mathrm{NiCl}_{2}-6 \mathrm{H}_{2} \mathrm{O}(5)$ & DMF (20/12) & 2a: 22 & n.d. \\
\hline 4 & - & $\mathrm{NiCl}_{2}-6 \mathrm{H}_{2} \mathrm{O}(5)$ & DMF (20/12) & $\mathbf{2 a}: 55(38)^{f}$ & n.d. \\
\hline 5 & \multicolumn{2}{|c|}{$(\mathrm{DME}) \mathrm{NiBr}_{2}(5)^{g, h}$} & THF $(60 / 24)$ & 2a: 29 & $21 \% \quad 49 \%$ \\
\hline 6 & \multicolumn{2}{|c|}{$(\mathrm{DME}) \mathrm{NiBr}_{2}(20)^{g, h, i}$} & THF $(60 / 1.5)$ & 2a: $81(56)^{f}$ & $9 \% \quad 8 \%$ \\
\hline 7 & \multicolumn{2}{|c|}{$(\mathrm{DME}) \mathrm{NiBr}_{2}(20)^{g, h, i}$} & $\operatorname{THF}(60 / 1.5)$ & 2b: 84 & $7 \% \quad 9 \%$ \\
\hline 8 & \multicolumn{2}{|c|}{$(\mathrm{DME}) \mathrm{NiBr}_{2}(5)^{g, h}$} & Toluene $(60 / 24)$ & 2a: Trace $^{j}$ & - \\
\hline 9 & \multicolumn{2}{|c|}{$(\mathrm{DME}) \mathrm{NiBr}_{2}(5)^{g, h}$} & Toluene/BMI ${ }^{k}(60 / 36)$ & 2a: Trace $^{j}$ & - \\
\hline 10 & DIPIMP & $\mathrm{NiCl}_{2}-6 \mathrm{H}_{2} \mathrm{O}(5)$ & Toluene/BMI ${ }^{k}(60 / 48)$ & 2a: 39 & n.d. \\
\hline 11 & MEEBI & $\mathrm{NiBr}_{2}-6 \mathrm{H}_{2} \mathrm{O}(5)$ & Toluene/BMI ${ }^{k}(60 / 1)$ & 2a: $91(65)^{f}$ & $4 \%$ \\
\hline 12 & MEEBI & $\mathrm{NiBr}_{2}-6 \mathrm{H}_{2} \mathrm{O}(5)$ & Toluene/BMI ${ }^{k}(60 / 1)$ & 2b: $85(53)^{f}$ & $10 \%$ \\
\hline 13 & MEEBI & $\mathrm{NiBr}_{2}-6 \mathrm{H}_{2} \mathrm{O}(2)$ & Toluene/BMI $^{k}(60 / 12)$ & 2a: $67(46)^{f}$ & $9 \% \quad 19 \%$ \\
\hline 14 & MEEMI & $\mathrm{NiBr}_{2}-6 \mathrm{H}_{2} \mathrm{O}(5)$ & Toluene/BMI ${ }^{k}(60 / 1)$ & 2a: $81(54)^{f}$ & $9 \%$ \\
\hline
\end{tabular}

${ }^{a} \mathbf{1}$ (1.0 mmol, 1a for run 1-6, 8-11, 13 and 14 or $\mathbf{1 b}$ for run 7 and 12 was employed), Zn (0.10 mmol), solvent (4 mL). For the structure of DIPIMP, (DME) $\mathrm{NiBr}_{2}$, MEEBI, MEEMI and BMI, see below. ${ }^{b}$ Ligand : $\mathrm{Ni}=1.2: 1.0$. ${ }^{c}$ Determined by ${ }^{1} \mathrm{H}$ NMR analysis. Diyne 1a was consumed, except for run 8 and 9. ${ }^{d}$ By-products (benzenes) consisted of 3, 4 and other compounds having a benzene skeleton. ${ }^{e}$ Based on the weight of separated polymer by column 5 chromatography. ${ }^{f}$ Isolated yield. ${ }^{g}$ Commercially available complex was used as received. ${ }^{h} \mathrm{THF}(10 \mathrm{ml})$. Equimolar amount of $\mathrm{H}_{2} \mathrm{O}$ with Ni was added. ${ }^{i} 40 \mathrm{~mol} \%$ of zinc was used. ${ }^{j}$ No reaction took place. ${ }^{k}$ Toluene : BMI $=20: 1$.

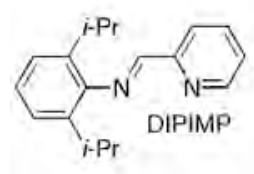

high yield, $81 \%$ and $84 \%$, respectively, but the production of polymeric material appeared to be unavoidable as evident from 10 our several attempts (run 6 and 7 ). ${ }^{\ddagger}$ Although the selectivity between COT 2 and benzene derivatives is important for attaining a good yield of $\mathbf{2}$, in all cases the polymeric product was produced. Minimizing polymer formation might also be an effective means to increase the yield of 2 . We assumed that these 15 side reactions may be proceeding through reactions of a single nickelacycle intermediate with diynes $\mathbf{1}$. If the reaction affording COT is proceeding through a mechanism involving the reaction between two nickelacycle intermediates as suggested by Wilke ${ }^{5}$ and Wender, ${ }^{6}$ introduction of a biphasic system for isolating the 20 catalyst into one phase, separating the catalyst from the substrate and maintaining high catalyst concentration throughout the reaction, may increase selectivity for the production of $\mathbf{2}$.

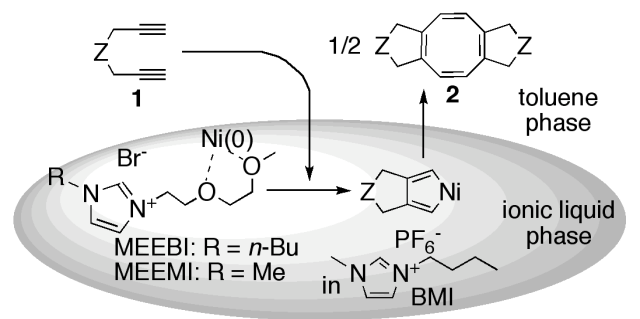

Fig. 2 Diremization of 1,6-diynes in a biphasic system.

25 To test this idea, we introduced a toluene/ionic liquid ${ }^{5}$ biphasic $^{2}$ system (Figure 2 and run 9-14 in Table 1). In a mixture of toluene and BMI (3-butyl-1-methyl-1H-imidazol-3-ium hexafluorophosphate) $(20 / 1, \mathrm{v} / \mathrm{v})$ as a solvent, the reaction in the presence of DIPIMP/ $\mathrm{NiCl}_{2}-6 \mathrm{H}_{2} \mathrm{O} / \mathrm{Zn}$ showed somewhat better

30 yield of $\mathbf{2 a}$ than that in THF (run 1 vs. run 10). Meanwhile, $\mathrm{NiCl}_{2}-6 \mathrm{H}_{2} \mathrm{O} / \mathrm{Zn}$ and $(\mathrm{DME}) \mathrm{NiBr}_{2} / \mathrm{Zn}$ reagents did not catalyze the reaction in this solvent system. To attain a more effective separation of the catalyst into the ionic liquid phase, we prepared imidazolium salts having a DME-like side chain, MEEBI (135 methoxyethoxyethyl-3-butyl- $1 H$-imidazol-3-ium bromide) and MEEMI (1-methoxyethoxyethyl-3-methyl-1H-imidazol-3-ium

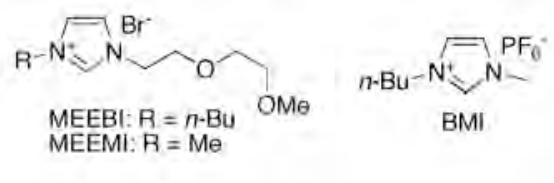

bromide), ${ }^{\S}$ and carried out the reaction with these salts as a ligand (Figure 2 and figures in Table 1).

As revealed in runs $11-14,5 \mathrm{~mol} \%$ of $\mathrm{MEEBI} / \mathrm{NiBr}_{2}-3 \mathrm{H}_{2} \mathrm{O}$ or 40 $\mathrm{MEEMI} / \mathrm{NiBr}_{2}-3 \mathrm{H}_{2} \mathrm{O}$ in toluene/BMI with $\mathrm{Zn}$ powder effectively dimerized 1 to 2 in good yield with high selectivity (run 11 and 12). Reducing the amount of ligand and nickel to $2 \mathrm{~mol} \%$ also afforded 2 in synthetically useful yield (run 13). The reaction under the conditions shown in run 11 provided COT $\mathbf{2 a}$ in $91 \%$ ${ }_{45}{ }^{1} \mathrm{H}$ NMR yield, the crude of which was subjected to column chromatography to remove the more polar trimer $\mathbf{4 a}$ and polymeric materials. Pure 2a was obtained in $65 \%$ isolated yield after the resulting solid of $\mathbf{2 a}$ was washed with hexane/EtOAc (5:1) to remove residual dimer $\mathbf{3 a}$.

50 Representative examples of dimerization of diynes to COTs under the reaction conditions shown in run 11 of Table 1 and their isolated yields are summarized in Figure 3. The reactions provided the corresponding COTs in good isolated yields, where reasonable functional group compatibility was observed.
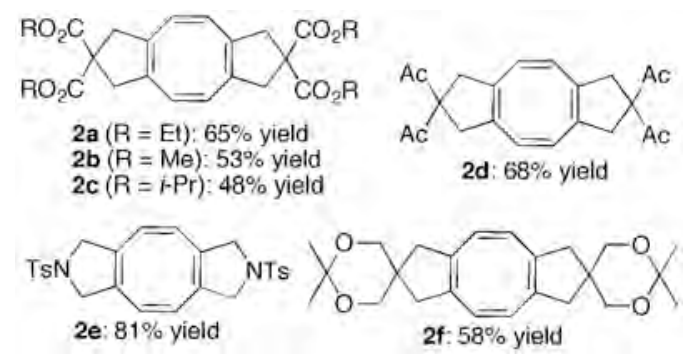

Fig. 3 Preparation of cyclooctatetraenes 3 by dimerization of diynes 2 .

Although all attempts to polymerize $\mathbf{2 a}$ and $\mathbf{2 e}$ to the corresponding 5 by ROMP with 1st- or 2nd-generation Grubbs $\mathrm{Ru}$-carbene catalysts and Ciba-ruthenium ${ }^{\circledR}$ under various reaction 60 conditions were unsuccessful, we were interested in the polymeric product of the present $\mathrm{Ni}$-catalyzed reaction of $\mathbf{1 a}$ as a polymer similar to $\mathbf{5}$. Thus, column chromatography of the crude residue of the reaction shown in run 1 of Table 1 separated less polar yellow- and polar deep orange-colored fractions, which ${ }_{65}$ were subjected to GPC (gel-permeation chromatography), TOF- 
MS, UV-vis, ${ }^{1} \mathrm{H}$ NMR and IR analyses (See Supplementary Information). From GPC (THF) analyses, $M \mathrm{w}$ of these fractions were calculated to be $1.2 \times 10^{3}$ and $5.1 \times 10^{3}$ for yellow- and deep orange-materials, respectively. Based on the analytical data of 5 these materials, the colored materials were characterized as polymers having the structure 6a (Figure 4).

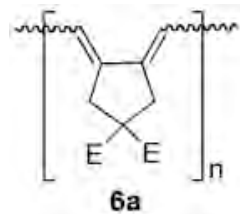

Fig. 4 Proposed polymer structure.

\section{Notes and references}

${ }_{25}{ }^{a}$ Department of Material \& Life Chemistry, Kanagawa University, 3-27-1 Rokkakubashi, Kanagawa-ku, Yokohama 221-8686, Japan. Fax: +8145 413 9770; Tel: +81 45481 5661; E-mail: okamos10@kanagawa-u.ac.jp ${ }^{b}$ New Products Development Division, Kanto Denka Kogyo Co., Ltd., 425 Kanai, Shibukawa, Gunma 377-0027, Japan.

$30 \dagger$ Electronic Supplementary Information (ESI) available: [details of any supplementary information available should be included here]. See DOI: $10.1039 / \mathrm{b} 000000 \mathrm{x} /$

* Although there is no description on polymeric product in the literature (ref 6), several experiments with $\mathbf{1 a}$ or $\mathbf{1 b}$ always gave us a mixture of $\mathbf{2}$, 35 benzenes $(\mathbf{3}, \mathbf{4})$ and polymeric material.

$\S$ MEEBI and MEEMI, which are liquid under ambient conditions, were prepared by the reaction of $\mathrm{N}$-methyl- or -butyl-imidazole with $\mathrm{BrCH}_{2} \mathrm{CH}_{2} \mathrm{OCH}_{2} \mathrm{CH}_{2} \mathrm{OMe}$ at $80{ }^{\circ} \mathrm{C}$. For other IL-tagged ligands and their use for catalysis in ILs, see ref $3 \mathrm{~d}$.

401 (a) E. K. van den Beuken, B. L. Feringa, Tetrahedron 1998, 54, 12985. (b) M. Kitamura, S. Suga, H. Oka, R. Noyori, J. Am. Chem. Soc. 1998, 120, 9800. (c) D. Allen Annis and Eric N. Jacobsen J. Am. Chem. Soc. 1999, 121, 4147. (d) M. Shibasaki, N. Yoshikawa, Chem. Rev. 2002, 102, 2187. (e) G. M. Sammis, E. N. Jacobsen, J. Am. Chem. Soc. 2003,

45 125, 4442. (f) G. M. Sammis, H. Danjo, E. N. Jacobsen, J. Am. Chem. Soc. 2004, 126, 9928. (g) F. X. Chen, H. Zhou, X. H. Liu, B. Qin, X. M. Feng, G. L. Zhang, Y. Z. Jiang, Chem. Eur. J. 2004, 10, 4790. (h) J.-A. Ma, D. Cahard, Angew. Chem. Int. Ed. 2004, 43, 4566. (i) Glenn M. Sammis, Hiroshi Danjo, and Eric N. Jacobsen J. Am. Chem. Soc.

50 2004, 126, 9929. (j) S. S. Kim, D. H. Song, Eur. J. Org. Chem. 2005, 1777. (k) Y. Shi, S. M. Peterson, W. W. Haberaecker III, S. A. Blum, J. Am. Chem. Soc. 2008, 130, 2168.

2 For example, see: X. Zheng, C. W. Jones, M. Weck, J. Am. Chem. Soc. 2007, 129, 1105.

553 (a) T. Welton, Chem. Rev. 1999, 99, 2071. (b) J. Dupont, R. F. de Souza, P. A. Z. Suarez, P. Chem. Rev. 2002, 102, 3667. (c) V. I. Parvulescu, C. Hardacre, Chem. Rev. 2007, 107, 2615. (d) R. Sebesta, I. Kmentová, S. Toma, Green Chem. 2008, 10, 484.

4 W. Reppe, O. Schlichting, K. Klager, T. Toepel, Justus Liebigs Ann. 60 Chem. 1948, 560, 1.

5 G. Wilke, Pure Appl. Chem. 1978, 50, 677.

6 P. A. Wender, J. P. Christy, J. Am. Chem. Soc. 2007, 129, 13402.

7 O. A. Scherman, I. M. Rutenberg, R. H. Grubbs, R. H. J. Am. Chem. Soc. 2003, 125, 8515.

658 (a) J. H. Rigby, N. C. Warshakoon, Tetrahedron 1997, 38, 2049. (b) C. J. Lawrie, K. P. Gable, B. K. Carpenter, Organometallics 1989, 8, 2274. (c) Y. Yamamoto, T. Ohno, K. Itoh, Chem. Eur. J. 2002, 8, 4734. (d) C. Chen, C. Xi, C. Lai, R. Wang, X. Hong, Eur. J. Org. Chem. 2004, 3, 647. (e) C. Wang, J. Yuan, G. Li, Z. Wang, S. Zhang, 70 Z. Xi, J. Am. Chem. Soc. 2006, 128, 4564. (f) P. A. Wender, M. P.
Croatt, B. Witulski, Tetrahedron 2006, 62, 7505 and references therein.

9 (a) N. Saino, D. Kogure, S. Okamoto, Org. Lett. 2005, 7, 3065. (b) N. Saino, D. Kogure, K. Kase, S. Okamoto, J. Organomet. Chem. 2006 , 691, 3129. (c) N. Saino, F. Amemiya, E. Tanabe, K. Kase, S Okamoto, Org. Lett. 2006, 8, 1439. (d) A. Goswami, T. Ito, S. Okamoto, Adv. Synth. Catal. 2007, 349, 2368.
In conclusion, we have demonstrated that a $\mathrm{Ni}$-catalyst with 10 use of an appropriate ligand and reaction media enables selective formation of COTs 2 and polymer of type 6 from 1,6-diynes. To immobilize the metal catalyst into an ionic liquid phase and control the reaction to be selective for COT formation, we have designed a reaction system consisting of a toluene/ionic liquid 15 biphasic solvent system and a ligand having an imidazolium substructure in the side chain. Ionic liquids have often been utilized for recycling metal-based catalyst and/or as alternatives to conventional solvents. Although examples that introduce a biphasic system that alters the reactivity and selectivity of the 20 catalysis have been studied, ${ }^{3}$ utilization of an IL/organic solvent biphasic system for controlling the reactions via a bimetallic intermediate, like the present case, has scarcely been reported. 